\title{
Histoire de l'architecture et des jardins du Japon pré-moderne
}

(Milieu du XVI ${ }^{\mathrm{e}}$ siècle - milieu du XIX ${ }^{\mathrm{e}}$ siècle)

Nicolas Fiévé

\section{(2) OpenEdition}

Journals

Édition électronique

URL : https://journals.openedition.org/ashp/1372

DOI : 10.4000/ashp.1372

ISSN : 1969-6310

Éditeur

Publications de l'École Pratique des Hautes Études

Édition imprimée

Date de publication : 1 octobre 2012

ISSN : 0766-0677

Référence électronique

Nicolas Fiévé, "Histoire de l'architecture et des jardins du Japon pré-moderne », Annuaire de l'École pratique des hautes études (EPHE), Section des sciences historiques et philologiques [En ligne], 143 | 2012, mis en ligne le 28 septembre 2012, consulté le 03 août 2021. URL : http://journals.openedition.org/ ashp/1372 ; DOI : https://doi.org/10.4000/ashp.1372 


\title{
HISTOIRE DE L'ARCHITECTURE ET DES JARDINS DU JAPON PRÉ-MODERNE
} (MILIEU DU XVI ${ }^{\mathrm{e}}$ SIÈCLE - MILIEU DU XIX ${ }^{\mathrm{e}}$ SIÈCLE)

\author{
Directeur d'études : M. Nicolas FIÉvé
}

Programme de l'année 2010-2011 : En suivant les seigneurs domaniaux : les grands jardins paysagers de l'époque d'Edo.

Les seigneurs domaniaux, nouveaux mécènes de l'art des jardins dans le Japon de l'époque d'Edo

Daimyō 大名, ou " grand nom », désigne au Japon depuis la période médiévale (1185-1573) un seigneur qui possède un fief, ryōchi 領地 ou han 藩, et qui est le vassal du gouvernement militaire, bakufu 幕府. À l'époque d'Edo (1603-1867), un « grand nom » doit avoir un fief dont la production annuelle de riz est évaluée à au moins 10000 koku石, un koku équivalant à la quantité de 180 litres de riz. Au début $\mathrm{du} \mathrm{XVII}^{\mathrm{e}}$ siècle, l'ensemble des revenus du pays donne le chiffre approximatif d'une production de trente millions de koku de riz. Le nouveau gouvernement des Tokugawa 徳川幕府, à la différence de ceux qui l'avaient précédé, a gardé une base territoriale importante : le shōgunat dispose d'un peu moins du quart de la production de l'ensemble du territoire, soit sept millions de koku, répartis en quatre millions pour le domaine du shōgun et trois millions pour les innombrables petits domaines attribués aux « hommes de la bannière », hatamoto 旗本, petits vassaux héréditaires des Tokugawa en charge des postes secondaires de l'administration. Les daimyō se voient attribués la part considérable de $75 \%$ des richesses, soit 22,5 millions de koku de riz, alors que les aristocrates n'en possèdent plus que $100000 \mathrm{koku}$, soit $0,3 \%$, répartis en $30000 \mathrm{koku}$ pour la maison impériale et $70000 \mathrm{koku}$ pour le reste des nobles. Les établissements religieux, temples bouddhiques et sanctuaires shintō, disposent quant à eux d'un revenu d'environ $400000 \mathrm{koku}$, soit 1,3\% de l'ensemble des richesses du pays.

Dans le nouveau système imposé par le gouvernement des Tokugawa, les daimyō sont classés selon leur origine. On distingue en haut de l'échelle politique les daimyō appartenant à la famille du shōgun ou assimilés à elle, que l'on nomme les «Trois maisons », Gosanke 御三家, trois lignées issues de fils cadets de Tokugawa Ieyasu 徳 川家康 (1542-1616), les maisons d'Owari 尾張, de Kii 紀伊 et de Mito水戸, aptes à fournir un successeur à la lignée des shōguns; les fudai daimyō 譜代大名, fidèles serviteurs du shōgun, descendants de vassaux héréditaires d'Ieyasu dès avant 1600 (Keichō 5), date de la bataille de Sekigahara 関ヶ原 et de la prise du pouvoir des Tokugawa; les « daimyō extérieurs », tozama daimyō 外様大名, issus d'anciennes familles et de seigneurs de la guerre de l'époque des luttes entre les provinces (1467-1573), mais devenus vassaux du shōgun après la bataille de Sekigahara. 
Les daimyō sont par ailleurs hiérarchisés suivant l'importance territoriale de leur fief et par le fait que certains possèdent un château, shiromochi 城持 ou jōshu 城主, et que d'autres en sont dépourvus, mujō 無城. Moins de trois cents seigneurs se partagent les trois quarts du territoire, avec des fiefs dont le revenu est compris dans une fourchette allant de dix mille à un million de koku. En haut de l'échelle, prennent place les seigneurs détenteurs de château et dont le fief couvre une ou plusieurs provinces, kuni$m o c h i$ 国持 ou kokushu 国主, comme le seigneur de la maison Maeda 前田家, du fief de Kaga 加賀藩, ou celui de la maison Shimazu 島津家, du fief de Satsuma 薩摩藩.

À côté des Trois maisons, on comptait à l'origine 37 familles de daimyō héréditaires, fudai daimyō, nombre qui passe progressivement à 145 , et moins d'une centaine de daimyō extérieurs. Dans ce dernier groupe, apparaissent les noms des plus anciennes maisons et les fiefs les plus vastes. Sur le plan économique, certains daimyō extérieurs comptent parmi les plus puissants du pays. À titre d'exemple, en 1664, la maison Maeda, dont le fief de Kaga comprend les provinces de Kaga, Noto 能登国 et Etchū 越中国, a un revenu estimé à 1030000 koku, la maison Shimazu, dont le fief de Satsuma réuni les provinces de Satsuma et d'Ōsumi 大隅国, est à la tête d'un revenu d'à peu près 730000 koku, alors que la maison Hosokawa 細川家, du fief de Kumamoto 熊本藩 (soit la plus grande partie des provinces de Higo 肥後国 et de Bungo 豊 後国), a, quant à elle, un revenu de 540000 koku.

Tous les puissants daimyō, tozama ou non, dépensèrent largement, dès le début de la période d'Edo, pour la construction de châteaux et de vastes villas de plaisance. Les anciens jardins de ces palais, ceux qui ont traversé l'histoire jusqu'à nos jours, comptent parmi les plus beaux parcs paysagers du pays : les trois maisons précédemment citées ont légué le parc Kenrokuen 兼六園 (10 hectares), à Kanazawa 金沢, dont les travaux de la partie basse ont été lancés dès 1676 (Enpō 4) par le $5^{\mathrm{e}}$ daimyō Maeda Tsunanori 前田綱紀 (1643-1724), le parc Sengan.en 仙窄園 (5 hectares), à Kagoshima, dont le $2^{\mathrm{e}}$ seigneur Shimazu Mitsuhisa 島津光久 (1616-1695) a entrepris les travaux en 1658 (Manji 1), et le parc Jüjuen 成趣園 (7,3 hectares) à Kumamoto, dont le $2^{\mathrm{e}}$ seigneur Hosokawa Tadatoshi 細川忠利 (1586-1641) a fait bâtir un premier pavillon de thé (Suizenji ochaya 水前寺御茶屋) en 1636 (Kan.ei 13), avant que son petit-fils, le $4^{\mathrm{e}}$ seigneur Hosokawa Tsunatoshi 細川綱利(1643-1714), ne fasse creuser les lacs, aménager les cours d'eau, édifier les montagnes artificielles et ne donne à ce splendide jardin paysager le nom de Jūjuen, d'après une poésie du lettré chinois Tao Yuanming 陶淵明 (365-427).

De leur côté, les Trois maisons, Owari (620000 koku), Kii (540000 koku) et Mito (240000 koku), jouissaient de fiefs dits « apparentés », shinpan 親藩, des fiefs confiés à des membres de la maison Tokugawa. Deux autres fiefs furent aussi désignés comme shinpan : Echizen 越前, donné à Yūki Hideyasu 結城秀康 (1574-1607), fondateur de la maison des Matsudaira d'Echizen 松平越前家, et celui d'Aizu 今津藩 (230000 koku en 1643), dans la province de Mutsu 陸奥国, confié en 1643 à Hoshina Masayuki 保科正之 (1611-1673), fondateur de la maison des Matsudaira d'Aizu 松平 今津家. Les daimyō de ces cinq domaines recevaient le traitement honorifique le plus élevé. Au XVIII ${ }^{\mathrm{e}}$ siècle, enfin, le $8^{\mathrm{e}}$ shōgun Tokugawa Yoshimune 徳川吉宗 (16841751) a organisé le système des « Trois hauts dignitaires », Sankyō 三卿, de la maison Tokugawa. À la différence des Trois maisons, les Trois hauts dignitaires, composées 
des trois familles Tayasu 田安家, Hitotsubashi 一橋家 et Shimizu 清水家, ne furent pas dotées de fiefs. Elles avaient mission d'assister le shōgun, voire de fournir un héritier si la lignée directe survenait à manquer.

Plusieurs jardins d'anciennes propriétés des « Trois maisons » ou des « Trois hauts dignitaires " ont subsisté : parmi ceux-ci, le jardin du château de Nagoya, Nagoya-jō teien 名古屋城庭園 (1615-1623 et 1716-1736), l'ancien château de la maison d'Owari détruit en 1945, mais dont subsistent $3500 \mathrm{~m}^{2}$ de jardins du palais Ni no maru goten 二の丸御殿, composés d'admirables assemblages de pierre réalisés au cours des années Genwa (1615-1623), qui furent remaniés en un jardin sec après l'ère Kyōhō (1716-1736); une partie du Yakusōen 薬草園 (dénommé de nos jours Oyakuen 御薬 園, 1,7 hect.), dans l'ancien fief d'Aizu, un jardin commencé en 1670 (Kanbun 10) par le $2^{\mathrm{e}}$ seigneur Matsudaira (Hoshina) Masatsune 正経 (1647-1681), mais dont l'aspect actuel remonte aux travaux de 1696 (Genroku 9), effectués par son successeur Matsudaira Masakata 正容 (1669-1731); le Kairakuen 偕楽園 de Mito (13 hect.), édifié en 1841 (Tenpō 12) par le 9e seigneur de Mito, Tokugawa Nariaki 徳川斉昭 (1600-1660).

Beaucoup d'autres propriétés ont aujourd'hui disparu, mais plusieurs sources en évoquent les apparences. Des plans et quelques rouleaux illustrés dépeignent les vues de jardins organisés autour de lacs et d'étangs : Owari-kō bessō michi no zu 尾張公別 荘道図 (Vues du cheminement à travers la résidence secondaire du seigneur d'Owari) [fin $\mathrm{XVIII}^{\mathrm{e}}$ s., archives Mitsui bunko 三井文庫], où sont réprésentés avec une grande précision les paysages, les assemblages de pierres et les édifices de la «résidence inférieure », shimoyashiki 下屋敷, du seigneur du fief d'Owari, dite Toyama-sō 戸山 荘, une résidence dont on possède par ailleurs la copie d'un plan réalisé entre 1751 et 1764; Yokuon.en shinkei zukan 浴恩園真景図巻 (Rouleau illustré des véritables paysages du Yokuon.en) [début XIX ${ }^{\mathrm{e}}$ s., Bibliothèque centrale de l'université de Tenri, Tenri daigaku fuzoku Tenri toshokan-zō 天理大学付属天理図書館蔵], une peinture de Hoshino Bunryō 星野文良 († 1846), qui montre avec force de détails les jardins de la résidence d'Edo de la famille Tayasu, un shimoyashiki, dont on connaît également une copie du plan du parc en 1840. L'étude de ces deux peintures naturalistes et de ces plans, introduite au cours de l'année, rend possible la reconstitution de l'espace architectural et paysager de ces sites.

Ce détour par une rapide présentation de la hiérarchie qui prévalait parmi les seigneurs donne la mesure de l'exceptionnelle assise économique des plus puissants d'entres eux. Une richesse qui leur a permis d'entreprendre sans relâche la construction de nombreux châteaux, de «villes bâties autour du château », jōka-machi 城下 町, de palais, de villégiatures, de parcs et de jardins, et d'inaugurer ainsi un développement des villes et de l'architecture tel que le pays n'en avait jamais connu, si ce n'est à l'époque ancienne (595-1185), mais sous une forme bien différente, il est vrai! Il en allait ainsi des nécessités de la vie politique et économique, mais aussi des modes du temps et de la recherche d'un plaisir personnel - ces palais et leurs innombrables pavillons ont été les lieux d'accueil où l'on recevait les autres seigneurs, leurs émissaires et les hommes célèbres de son temps.

Sur l'ensemble de l'époque d'Edo, ce sont 434 familles qui donnèrent près de 3500 daimyō. L'installation définitive de la paix, pour plus de deux siècles, adjointe d'une bonne gestion des domaines, dont la productivité augmente en début de période, 
a scellé l'écrasante suprématie économique des Tokugawa et des seigneurs domaniaux sur les nobles et la Cour impériale. De ce point de vue, le XvII ${ }^{\mathrm{e}}$ siècle a inauguré une ère nouvelle, dans laquelle les daimyō se sont définitivement substitués aux nobles de Kyōto et aux monastères bouddhiques pour assumer un rôle de mécènes dans les domaines de l'art, de l'architecture et du paysagisme, un rôle que moines et nobles avaient encore en partie assumé aux époques précédentes à Kyōto.

Les principaux daimyō, ceux possesseurs de province ou ceux appartenant aux Trois maisons, entretenaient trois palais à Edo : une « résidence supérieure », kamiyashiki 上 屋敷, c'est-à-dire la demeure principale (hontei 本邸) du seigneur et de son épouse, une « résidence intermédiaire », nakayashiki 中屋敷, où vivaient le daimyō retiré et les héritiers de la maison, une « résidence inférieure », shimoyashiki 下屋敷, une villa secondaire (bettei 別邸 ou bessō 別荘), lieu de villégiature et de repos, où demeurait souvent une partie de la suite et de l'équipage. Les daimyō moins aisés ne possédaient que deux résidences, kamiyashiki et shimoyashiki. On notera que dans les provinces la situation était le plus souvent la même, ce qui nécessitait l'entretien simultané de quatre, voire de six résidences.

À Edo, les palais principaux (kamiyashiki) se situaient à proximité du château du shōgun, et occupaient des superficies moins vastes que les villas de plaisance (shimoyashiki), édifiées dans des quartiers périphériques, en bordure de campagne, et dont la superficie pouvait atteindre une dizaine d'hectares. Le parc Rikugien 六義園, qui fut tout d'abord le shimoyashiki de la maison Maeda, avant que le fudai daimyō Yanagizawa Yoshiyasu 柳沢吉保 (1658-1714), seigneur du fief de Kawagoe 川越 藩 dans la province de Musashi 武蔵国, entre en sa possession en 1695 (Genroku 8 ) et le remanie entièrement lui donner son aspect actuel, est une propriété paysagère de 8,8 hectares de superficie, et sans doute l'une des plus intéressantes du point de vue de l'histoire des jardins qui subsiste encore de nos jours à Tōkyō. Le Kiyosumi teien 清澄庭園, ancien shimoyashiki (vers 1716-1735) des seigneurs Kuze 久 世, du fief de Sekiyado 関宿藩 dans la province de Shimo.usa 下総国, atteint quant à lui 8,1 hectares de superficie et reste particulièrement remarquable pour ses assemblages de pierres de grande dimension. Dans les provinces, le kamiyashiki était bâti dans l'enceinte du château seigneurial, alors que le shimoyashiki était installé dans un faubourg ou une campagne proche, là où l'on trouvait les paysages naturels les plus pittoresques : en bordure de la mer à Wakayama, le long d'une rivière à Hiroshima 広 島 ou au pied de montagnes boisées à Takamatsu 高松. À Kagoshima 鹿児島, la villégiature de la maison Shimazu est à flanc de montagne et en bordure de mer, face au somptueux paysage du volcan en activité de l'île Sakura-jima 桜島.

Ces lieux de villégiature implantés sur de vastes parcs comprenaient les habitations du seigneur et des membres de sa famille, les quartiers de la suite et du personnel. Le parc-promenade était le plus souvent de type kaiy $\bar{u}$-shiki teien 回遊式庭 園, un «jardin de plaisance autour d'une pièce d'eau », et reproduisait en miniature des paysages renommés du pays ou de la Chine, parmi lesquels on aimait se déplacer comme lors d'un voyage. Les jardins étaient équipés de kiosque (azumaya 東屋, 亭), de pavillons de thé (chashitsu 茶室) et autres « pavillons de style raffiné » (sukiya 数 寄屋), où l'on pouvaient se détendre et admirer les paysages et le passage des saisons, que des champs d'érables (aux feuilles rougies pendant l'automne) et de cerisiers (à 
la floraison printanière) soulignaient ostensiblement. Les parcs comprenaient souvent un ou plusieurs petits temples bouddhiques et sanctuaires shintō, un champ de courses, parfois une zone réservée à la chasse aux canard, des embarcadères sur les étangs et les lacs. Fruit du mélange des arts et des techniques de tout le pays, on y trouvait incontestablement ce que l'époque avait produit de plus raffiné en matière d'architecture palatiale et de villégiature, de jardins et de reproductions de paysages célèbres.

Alors que, dès les premières années du XVII ${ }^{\mathrm{e}}$ siècle, le bakufu a œuvré à mettre au pas la Cour et les nobles - désormais privés de toute participation à la vie politique et économique du pays -, le shōgun mettait en place dans le même temps un système de compensation par lequel il entreprenait le financement des travaux de construction d'un imposant palais impérial, à Kyōto, orné d'un jardin-paysage de $8000 \mathrm{~m}^{2}$, et offrait dans le même temps des cadeaux aux membres de la maison impériale qui lui avaient rendu des services. Ainsi, le prince Hachijō no miya Toshihito 八条宮智 仁 (1579-1629) et son fils Toshitada 智忠 (1620-1662) réussirent en partie à financer ce qui demeure sans doute la plus belle villégiature de l'histoire de Kyōto : le palais détaché de Katsura, Katsura rikyū 桂離宮, dressé un parc d'une superficie de 5,6 hectares (Katsura rikyū est une désignation moderne; on parle dans les sources de « maisons de thé de Katsura », Katsura no chaya 桂の茶屋). Et il en allait de même pour les édifices et les jardins de plusieurs monastères de premier rang, dont la puissance et l'assise économique avait décliné au fil des conflits de la période médiévale. Audelà de leurs propres jardins et palais qu'ils développaient sur des échelles nouvelles, les seigneurs domaniaux devenaient ainsi dans le même temps les mécènes des palais de la Cour, de certains nobles et de monastères. Un phénomène qui a contribué à la diffusion de nouveaux styles architecturaux et paysagers dans l'ensemble des élites du pays.

Il faut ici rappeler que les époques précédentes, marquées par des siècles de guerre civile, n'avaient pas permis aux seigneurs de la guerre autres que les puissants shōguns de promouvoir des palais bâtis au milieu de vastes jardins de plaisance. Hormis les palais shōgunaux de Kyōto et deux retraites aménagées par les shōguns Ashikaga, la presque totalité des jardins renommées de l'époque de Muromachi (1336-1573) avaient été l'œuvre de monastères bouddhiques, et, plus modestement, de la maison impériale ou de nobles - les aristocrates ayant été depuis l'époque ancienne les promoteurs de l'art des jardins. Pour l'essentiel, ces jardins avaient été conçus à Kyōto, berceau incontestable des paysagistes japonais et de leur savoir-faire, mais avaient consisté en des dispositifs de petite superficie, édifiés autour, ou entre, les bâtiments d'une habitation, et ne dépassant pas quelques centaines de mètres carrés. Lorsque la maison Ashikaga est au sommet de sa puissance, soit à l'époque du $3^{\mathrm{e}}$ shōgun Ashikaga Yoshimitsu 足利義満 (1358-1408), le très renommé palais des fleurs, Hana no gosho 花御所, le plus vaste de son temps bâti intra muros à Kyōto, ne couvre qu'une superficie de deux quartiers, soit moins de 2,5 hectares, dont seule une modeste partie était occupée par les jardins. Tous les autres palais des shōguns Ashikaga et le palais impérial lui-même ne couvraient tout au plus qu'un quartier, soit un hectare en moyenne de superficie, une surface occupée pour l'essentiel par des édifices qu'agrémentaient des cours et des jardins de taille modeste. Lorsque, dès le début de l'époque d'Edo, les seigneurs entreprennent la construction de vastes parcs-promenades d'une 
dizaine d'hectares de superficie, ils imposent alors la conception de nouveaux dispositifs paysagers jusqu'alors inconnus : représentations des saisons sous formes de champs monoculture, apparition de vastes étendues couvertes d'herbe (qui évoquent les pelouses occidentales), chapelets de paysages miniatures qui constituent des ensembles cohérents, comme au Jüjuen de Kumamoto, où ce sont divers sites de la route Tōkaidō 東海道, celle qui relie Kyōto à Edo, que l'on peut contempler en se déplaçant dans le paysage.

\section{Paysagistes professionnels et manuels sur l'art des jardins}

Le plus grand nombre des seigneurs domaniaux habitaient les provinces, parfois bien loin de Kyōto, et les shōguns Tokugawa résidaient à Edo, soit à environ 400 kilomètres de la capitale. L'époque d'Edo fut ainsi celle d'une ample circulation des hommes et, pour le sujet qui nous intéresse, des techniques et des savoirs relatifs à l'art des jardins et à l'architecture. Un mouvement qui se faisait dans un premier temps de Kyōto vers le reste du territoire, mais aussi, dès la fin du XVII ${ }^{\mathrm{e}}$ siècle, des lointaines provinces vers Edo et, par contrecoup, d'Edo vers Kyōto. Bien que l'archipel ait été une longue terre de 1800 kilomètres, la distance, tant physique que temporelle, ne semble pas avoir été un obstacle à la diffusion des motifs fondamentaux du jardin et de l'habitat des élites. Si différences stylistiques il y avait, celles-ci n'étaient guère été le fruit d'un régionalisme, mais plutôt la conséquence des changements de mode d'une époque.

Il faut rappeler que l'établissement du système dit de « venir rendre ses devoirs [au shōgun] », sankin kōtai 参勤交代, a été l'un des vecteurs les plus efficaces de la diffusion des arts et des connaissances dans tous les domaines du savoir. Instauré par le $3^{\mathrm{e}}$ shōgun Iemitsu 家光 (1604-1651), la décision est notée dans un amendement de 1635 (Kan.ei 12) aux règlements pour les daimyō. Selon l'article 2, tous les seigneurs, petits ou grands, étaient tenus de résider à Edo, la moitié de l'année pour les ceux du Kantō 関東, plus proches d'Edo, ou une année sur deux pour les autres. Pendant leur absence de la capitale, les daimyō devaient laisser leur famille à Edo - otages du pouvoir central, en quelque sorte. L'institutionnalisation de ce système permettait aux Tokugawa d'avoir les seigneurs sous surveillance la moitié du temps et de leur imposer double dépense pour la nécessité d'entretenir une double demeure, à la fois dans leur fief et à Edo. En conséquence, le personnel de toutes les maisons avait ainsi l'occasion de vivre à Edo et donc de ne pas réduire son champ de vision aux limites étroites de sa province d'origine.

On sait que certains daimyō sont montés à la ville shōgunale accompagnés de conducteurs de travaux, de jardiniers et d'horticulteurs venus de leur province, afin que ces derniers réalisent les jardins de leurs nouveaux palais. Un homme comme Kobori Masakazu 小堀政一 (1579-1647), resté célèbre sous le nom de Kobori Enshū 遠州 après sa nomination au poste de gouverneur de la province de Tōtōmi 遠江国 (le nom «Enshū » désigne cette province), avait reçu en 1606 (Keichō 11) la charge de préfet des travaux, sakuji bugyō 作事奉行, du palais de l'empereur retiré (Sentō gosho 仙洞御所). Repéré par ses pairs pour ses connaissances en matière d'architecture et de paysagisme, apprécié depuis son travail d'agrandissement du château de Nijō (Nijojōō 
二条城) et de ses jardins, des travaux entrepris à l'occasion de la visite de l'empereur Gomizunoo 後水尾天皇 (r. 1611-1629), Kobori Enshū a été sollicité tout au long de sa vie par de puissants seigneurs, afin qu'il leur fît bénéficier de ses talents et de son goût, si reconnaissables dans la conception de ses jardins. C'est ainsi que Mori Nagatsugu 森長継 (1610-1698), le $2^{\mathrm{e}}$ seigneur du fief de Tsuyama 津山藩, dans la province de Mimasaka 美作国, lui demanda de participer à la conception d'un jardin de plaisance qui s'inspirait de celui du palais impérial de Kyōto. Ainsi naquit le Shūrakuen 衆楽園, une demeure secondaire jadis de 7,4 hectares, dont il subsiste encore la partie orientale, composée de 2,8 hectares de jardins paysagers disposés autour d'un étang.

Une homme comme Ueda Sōko 上田宗箇 (1563-1650), Kamemaru 亀丸 de son prénom de naissance, fut également sollicité par de grands daimyō pour la conception de jardins. Ce guerrier, qui avait servi Toyotomi Hideyoshi 豊臣秀吉 (1537-1598) avant la bataille de Sekigahara et qui est entré par la suite au service du clan Asano 浅 野氏, avait étudié le thé comme son condisciple Kobori Enshū avec les maîtres les plus renommés de son temps : Sen no Rikyū 千利休 (1522-1591), puis Furuta Oribe 古田 織部 (1544-1615). Sōko a conçu, au cours des années Genwa (1615-1623), le jardin du Ni no maru goten二の丸御殿 du château de Nagoya $\left(3500 \mathrm{~m}^{2}\right)$, celui du Ni no maru goten du château de Wakayama $\left(8500 \mathrm{~m}^{2}\right)$ et le jardin du château de Tokushima 徳島城 $\left(5025 \mathrm{~m}^{2}\right)$. Des compositions réalisées toutes les trois dans le style original de l'école Kyokukan, Kyokukan-ryū 玉澗流, une tradition paysagère qui tire son nom du peintre chinois Ruofen (jap. Jakubun) 若芬 (1127-1279), dit Yujian (Kyokukan en japonais). Moine et peintre de la fin de la dynastie des Song du Sud et du début de celle des Yuan, Yujian avait peint un style de montagnes et de roches particulièrement escarpées qu'Ueda Sōko aimait représenter dans ses jardins (Yujian reste célèbre au Japon notamment pour une vue du Mont Lu 盧山, exécutée d'après un poème de Li Bai 李 白 (701-762), Lushan guanbao [jap. Rōzan kanbaku] 盧山観瀑 [La chute d'eau vue $d u$ Mont Lu]). Dans le jardin du Ni no maru goten (le palais de la Deuxième enceinte) du château de Nagoya, Sōko symbolise à l'aide de roches de grande dimension une gorge escarpée, coincée entre deux montagnes et que traverse un haut pont de pierre, dont l'effet pictural est des plus saisissants malgré un dispositif qui ne dépasse guère trois mètres de hauteur. À l'époque de cette réalisation audacieuse, le daimyō Asano Nagaakira 浅野長晟 (1586-1632) dirigeait le fief de Wakayama, avant qu'il ne reçût en 1619 (Genwa 5) celui de Hiroshima. Le fief de Kishū 紀州藩 (Wakayama), qui comprend la province de Kii 紀伊国 et la moitié sud de la province d'Ise 伊勢国, un ensemble d'un revenu annuel de $540000 \mathrm{koku}$, revient alors à Tokugawa Yorinobu 徳 川頼宣 (1602-1671) et le jardin du Nishi no maru (« enceinte de l'Ouest») est alors entièrement refait cette année-là. Ueda Sōko utilise alors une dénivellation naturelle $\mathrm{du}$ terrain d'une vingtaine de mètres pour réaliser une gorge profonde, au fond de laquelle se jette un cour d'eau, tel un torrent de montagne. Un cheminement escarpé permet au visiteur de descendre le long de la montagne, de franchir un pont au-dessus d'une ravine, d'enjamber l'écoulement d'une chute d'eau, avant d'arriver au fond d'une combe encaissée entre les escarpements rocheux et là, debout sur des pierres plates placées au milieu d'un lac, de pouvoir contempler la verticalité grandiose de ce paysage imaginaire qui rappelle la Chine. Après avoir reçu le fief de Hiroshima, un fief d'un revenu de $426000 \mathrm{koku}$ qui couvre la province d'Aki 安芸国 et la moitié de 
celle de Bingo 備後国, le seigneur Asano Nagaakira demande à Sōko, son vassal, de réaliser le parc de sa résidence secondaire, située à 400 mètres environ du château de Hiroshima, en bordure de la rivière Kyōbashi. Là, dès 1620 (Genwa 6), Sōko dirige les travaux du Shukkeien 縮景園, un vaste jardin de quatre hectares, organisé autour d'un étang et pour lequel il conçoit aussi les pavillons de thé. Un parc paysager de quatre hectares que remanie, au cours des années 1783 (Tenmei 3) et 1788 (Tenmei 8), Shimizu Shichirō.uemon 清水七郎右衛門, un maître jardinier invité de la capitale, Kyōto, alors située à plus de 350 kilomètres de routes qu'un homme du peuple comme un jardinier parcourait à pied. Ces paysagistes étaient des hommes que les seigneurs rétribuaient pour leur savoir et leur travail d'administrateur des travaux, une nouveauté dans un domaine artistique où jusqu'alors les aristocrates de Kyōto avaient conçus eux-mêmes les paysages qu'ils faisaient représenter dans leurs jardins.

Nonobstant, ce que pouvaient financer les hommes les plus influents de leur temps n'était pas à la portée de seigneurs ou de guerriers de rang plus modeste. En période de développement urbain et de circulation des hommes, l'écrit, sous la forme de manuel de composition des jardins, devient un vecteur non négligeable de la diffusion des connaissances en matière de paysage et d'horticulture. Dans ce domaine, deux familles de documents ont été distingués : des manuscrits, le plus souvent des rouleaux, dont quelques-uns sont antérieurs à l'époque d'Edo. Il s'agit de pièces uniques qui demeurent longtemps dans les mains d'un même propriétaire, des œuvres qui, malgré quelques copies, font l'objet d'une diffusion restreinte et réservée aux élites. Des livres imprimés, d'autre part, de plus en plus nombreux au fur et à mesure que l'on avance dans la période, largement vulgarisés dans l'ensemble du pays. On en recense une vingtaine de titres principaux, le plus souvent des livres illustrés. Restituant l'image des paysages artificiels, des arrangements de pierres et des collines, de l'implantation et de la forme des arbres, l'iconographie de ces ouvrages a contribué à la diffusion de formes stéréotypées du paysage, comme ce fut le cas au début du $\mathrm{XVII}^{\mathrm{e}}$ siècle avec la publication du Tsukiyama teizo-den 築山庭造伝 (Transmission sur les styles de jardins en montagnes artificielles), un ouvrage majeur pour l'histoire des jardins d'Edo, rédigé en 1735 (Kyōhō 20) par Kitamura Enkinsai 北村援琴 齋 (dates inconnues). Ce manuel emprunte l'essentiel de son texte à des traités plus anciens, comme le Saga-ryū niwa kohō hiden no sho 嵯峨流庭古法秘伝之書 (Livre des traditions secrètes de l'école Saga sur les anciennes techniques de composition des jardins, 1395 [Ōei 2]) ou le Tsukiyama sansui-den 築山山水伝 (Transmission sur les paysages de montagnes artificielles, 1723 [Kyōhō 8]). S'il n'innove pas, la large diffusion dont il est l'objet a eu un impact certain sur les pratiques, notamment dans les provinces éloignées de la capitale. De même, la version complétée de ce livre qu'édite en 1828 (Bunsei 11) Ritōken Akisato 離島軒秋里 (?-1830?), adjointe d'une seconde partie rédigée de la main de ce dernier, demeure sans aucun doute le plus célèbre des ouvrages sur les jardins de la fin de la période, tant par l'ampleur de sa diffusion, que par l'originalité de son contenu. On ne connaît à peu près rien de son auteur, ni de la formation qu'il aurait pu recevoir. Pourtant, la présentation qu'il donne des paysages a façonné durablement l'art des jardins moderne du Japon. L'ouvrage innove par sa présentation des styles shintai 真体, gyōtai 行体 et sōtai 草体, dont le vocable est hérité de la calligraphie chinoise - style régulier, zhen, intermédiaire, xing, cursif, cao - des 
différentes catégories de paysages en collines artificielles (tsukiyama sansui 築山山 水). Il présente en outre les typologies des jardins de courettes intérieures (tsubo-niwa 坪庭) et des jardins de thé (chatei 茶庭), comme celles d'éléments de mobilier de jardin : vasques à eau, dites «bols pour se rincer les mains » (chōzu-bachi 手水鉢), lanternes de pierre (seki tōrō 石燈籠). Une présentation de plusieurs jardins renommés de Kyōto, dont les exemples n'avaient pas été inclus dans le Miyako rinsen meishō-zue 都林泉名勝図会 (Récit illustré sur les jardins renommés de la capitale, 1799), un autre ouvrage d'Akisato qui fut un succès d'édition, complète cette présentation singulière des jardins de la période.

Il faut ici souligner qu'en dépit d'un assez grand nombre de titres nouveaux, rares sont les textes sur les jardins de l'époque d'Edo vraiment originaux. Un examen attentif des sources révèle une majorité de copies partielles ou intégrales des ouvrages les plus anciens - et cela qu'il s'agisse de manuscrits ou de livres imprimés. Que des textes vieux, parfois, de plusieurs siècles aient été remaniés, édités et présentés comme des nouveautés est un phénomène qui, dans une certaine mesure, a également contribué à la relative homogénéité des pratiques.

Il n'est pas fortuit que les deux plus anciens manuscrits connus sur l'art des jardins, le Sakutei-ki 作庭記 (Notes sur la composition des jardins) [XI ${ }^{\mathrm{e}}$ siècle] et le Senzui narabi ni yagyō no zu 山水並野形図 (Illustrations des paysages et des formes de plaines) [période médiévale, antérieur à 1466], soient passés des mains de nobles de Cour résidant à Kyōto, à celle de la puissante maison Maeda du fief de Kaga, quelques temps seulement avant 1676 (Enpō 4), date du début des travaux de la partie basse des jardins de l'enceinte extérieure du château de Kanazawa, au cours desquels le $5^{\mathrm{e}}$ daimyō Maeda Tsunanori fait creuser l'étang Renchitei 蓮池庭 et installer la grande chute d'eau que l'on peut aujourd'hui encore y admirer. Le jardin a été agrandi tout au long de l'époque d'Edo, jusqu'à devenir à la fin de la période le très renommé Kenrokuen 兼六園, un parc de dix hectares désigné depuis lors comme l'un des trois plus renommés du Japon. La plus ancienne copie du Sakutei-ki, dite Tanimura-bon 谷村本 (du nom d'une famille de Kanazawa à laquelle elle appartient aujourd'hui), est datée de 1289 (Shōō 2). Propriété de la famille noble des Fujiwara 藤原 durant le Moyen Âge, elle est transmise à la maison Maeda à l'époque de Tsunanori, alors que ce dernier entre en possession, dans le même temps, du plus ancien manuscrit connu du Senzui narabi ni yagyō no zu, une copie de 1466 (Bunshō 1), qui est demeurée la propriété de la maison Maeda jusqu'à la période contemporaine. Un document aujourd'hui conservé au Sonkei-kaku bunko 尊経閣文庫, archives qui rassemblent les fonds des anciennes bibliothèques de la maison Maeda.

Le transfert d'œuvres d'une telle valeur pour l'histoire des jardins, produites à l'origine par des nobles et réservées à leur propre usage, s'il révèle l'hégémonie des daimyō sur les nobles de Cour, il témoigne aussi du vif intérêt que ces grands seigneurs portaient aux anciens savoir-faire de la noblesse de Kyōto en matière de paysage. 